\title{
Periodic analytic approximate solutions for the Mathieu equation
}

\author{
M. Gadella ${ }^{\dagger}$, H. Giacomini ${ }^{\ddagger}$, L.P. Lara* \\ ${ }^{\dagger}$ Departamento de Física Teórica, Atómica y Óptica and IMUVA Universidad de \\ Valladolid, Paseo Belén 7, 47011 Valladolid, Spain, manuelgadella1@gmail.com. \\ ${ }^{\ddagger}$ Laboratoire de Mathématique et Physique Théorique, CNRS UMR 7350, Faculté des \\ Sciences et Techniques - Université de Tours, Parc de Grandmont 37200 Tours-France, \\ Hector.Giacomini@lmpt.univ-tours.fr. \\ *Departamento de Física, FCEIA, UNR, Av. Pellegini 250, 2000 Rosario, Argentina, \\ lplara@fceia.unr.edu.ar.
}

\begin{abstract}
We propose two methods to find analytic periodic approximations intended for differential equations of Hill type. Here, we apply these methods on the simplest case of the Mathieu equation. The former has been inspired in the harmonic balance method and designed to find, making use on a given algebraic function, analytic approximations for the critical values and their corresponding periodic solutions of the Mathieu differential equation. What is new is that these solutions are valid for all values of the equation parameter $q$, no matter how large. The second one uses truncations of Fourier series and has connections with the least squares method.
\end{abstract}

Keywords:

Mathieu equation, a modified harmonic balance method, leasts squares.

\section{Introduction}

The harmonic balance method $(1 ; 2 ; 3 ; 4)$ has been designed in order to obtain analytic approximations to periodic solutions for certain type of ordinary differential equations, linear or not. In this paper, we introduce an improved version of it and we test it in the Mathieu equation, in order to compare its efficiency with respect other methods and more specifically of the least square method. Henceforth, we shall denote it as the "Modified Harmonic Balance Method". 
The Mathieu equation is the simplest non-trivial type of Hill equation. This is a second order linear differential equation of the following type:

$$
\frac{d^{2} y(x)}{d x^{2}}+\left(A_{0}+\sum_{n=1}^{\infty} A_{n} \cos (2 n x)+\sum_{m=1}^{\infty} B_{m} \sin (2 m x)\right) y(x)=0 .
$$

This expression comes from a general linear second order equation of the type

$$
y^{\prime \prime}(x)+f(x) y(x)=0,
$$

in which we have spanned $f(x)$ into Fourier series. Equation (1), which is often called the general Hill equation, is intractable in general terms. In order to study tractable approximations to (1), we must truncate the series involved in the equation. In general, one chooses $B_{1}=B_{2}=\cdots=0$. Then, we say that the Hill equation is of order $n-t h$, if $A_{n} \neq 0$ and $A_{n+1}=A_{n+2}=\cdots=0$. Hill equations of first and second order are known as Mathieu and WhittakerHill equations, respectively $(5 ; 6 ; 7 ; 8)$. Further order Hill equations may be of interest in physics (9).

It is customary to write the Mathieu equation on the following form:

$$
y^{\prime \prime}(x)+(r-2 q \cos (2 x)) y(x)=0,
$$

where $A_{0}=r$, which is often called the characteristic value or eigenvalue, has to be determined through given boundary conditions, and $A_{1}=-2 q$, which is a fixed data. The solution $y(x)$ for a given $r$ is usually called the eigenfunction. Generally speaking, one looks for periodic solutions of (3), with boundary conditions at two finite fixed points, say 0 and $p$, given on the form $y(0)=y(p)$ and $y^{\prime}(0)=y^{\prime}(p)$, where $p$ is the period. This is certainly a particular case of a Sturm-Liouville problem.

The properties of the Mathieu equation (3) has been extensively studied $(10 ?)$. It has been established the existence of four countable sets of values for the characteristic value $r$, for which there exists periodic solutions, with period either $\pi$ or $2 \pi$. These four sets are associated to solutions that admit a series expansion on either even functions of the type $\cos (2 k) x$ or $\cos (2 k+1) x$, or odd functions of the form $\sin (2 k x)$ or $\sin (2 k+1) x$. Obviously, if $q=0$, the characteristic values are $r=m^{2}, m=1,2,3, \ldots$ and the solutions are $\cos (m x)$ and $\sin (m x)$. For $q \neq 0$, there is only one periodic solution for each characteristic value, being the second solution non-periodic. See also (11). 
The Sturm-Liouville problem associated with (1) has been solved by either methods based in Fourier series techniques $(12 ; 13 ; 14 ; 15)$ or by Taylor expansions $(10 ; 1)$. These methods require of numerical computations and they are usually valid for $|q|<1$ only. It seems that it would be desirable to have a method for solutions for large values of $|q|$.

Analytic approximate methods are usually one of the best tools to attack this kind of Sturm Liouville problems. In order to approximate periodic solutions, the harmonic balance method has been widely used $(10 ; 1)$, either for linear or non-linear ordinary differential equations. Here, we do not consider the non-linear case and for the Mathieu equation, we propose an analytic approximate method that provides both the critical value $r$ as well as the approximate periodic solution, which is valid even for large values of $|q|$. In few words, we obtain $r$ as solutions of some algebraic equations. No integrations, Taylor expansions nor complex manipulations with matrices are needed. Our method is conceptually simple and has been inspired in the harmonic balance method.

We must stress that the modified harmonic balance method is in principle applicable to other Hill equations beyond the Mathieu equation and eventually other type of equations admitting periodic solutions. We have focused our calculations in the Mathieu equation to test our preliminary results. Our modification avoids completely certain complications of the standard harmonic balance, as presented in (1), like the need of solving non-homogeneous differential equations with increasing complexity when more terms are included. In our modified harmonic balance, we just need to solve algebraic equations.

We should compare the results obtained by this modified harmonic balance with those got by more traditional methods, like the least square method. The results are essentially similar with degree of accuracy in both methods. Nevertheless, ours have the advantages of being simpler and easier to use by a computer and the least square method is more complicated to be practically implemented. For instance, the polynomial giving the approximate characteristic values is much simpler in our modified harmonic balance than in the least square method, as shown in subsection 4.1. This means, in particular, that the degree of algebraic equations is definitively smaller in our method as compared with the least squares. Note that the latter also gives spurious imaginary parts for roots.

The present article is organized as follows: In section 2, we introduce our modification of the harmonic balance method and give some numerical 
results. In section 3, we apply the method to a Mathieu equation with a purely imaginary parameter. In section 4 , we adapt the least square method to our situation. The results obtained by least squares are similar, although one needs both more precision and terms to obtain the same accuracy. In a limit sense, we show that the modified harmonic balance and the least square method are equivalent. We have written a section with concluding remarks plus an appendix on the relation between periodicity and parity of solutions for the general Hill equation.

\section{A modification on the Harmonic balance method}

Since our method has been inspired in the usual harmonic balance method, it could be interesting to recall the latter before a discussion of our ideas. Then, we first add here an introductory subsection with some comments on the harmonic balance, which will be also appropriate to justify further analysis. Then, our method will be introduced in the second subsection.

However, one should be aware of one important difference between the harmonic balance and our method. While the former relies on Taylor expansions on $q$, ours does not.

\subsection{The usual harmonic balance method}

We are looking for periodic solutions for (2) determined by the boundary conditions $y(0)=y(2 \pi)$ and $y^{\prime}(0)=y^{\prime}(2 \pi)$. In this case, the harmonic balance method, proposes the following type of solutions (1):

$$
r_{m}=m^{2}+\sum_{k=1}^{\infty} \alpha_{k} q^{k}, \quad Y_{m}(x)=\cos m x+\sum_{k=1}^{\infty} q^{k} c_{k}(x) .
$$

Note that if $q=0$, then, $r_{m}=m^{2}$ and $Y_{m}(x)=\cos m x$ give an even periodic solution, so that the Ansatz for $Y_{m}(x)$ is intended to construct even solutions. A similar Ansatz can be provided to construct odd solutions by replacing $\cos m x$ by $\sin m x$. The coefficients $\alpha_{k}$ and the functions $c_{k}(x)$ are indeterminate. To obtain them we have to use (4) in (1). The result is a system of second order non-homogeneous linear differential equations, which is in principle infinite, since there is an infinite number of $c_{k}(x)$. Nevertheless, in practice we produce an approximation by truncating the series (4), so that the number of $c_{k}(x)$ to be determined, and henceforth the number of equations, is finite albeit large. The inhomogeneous terms produce certain 
type of complications which could be overcome by a proper choice of the coefficients $\alpha_{k}$.

For $m=1$ one finds the following series for $r_{1}$ :

$$
r_{1}=1+q-\frac{q^{2}}{8}+\ldots
$$

If instead, we want odd solutions, $Y_{m}(x)$ in Ansatz (4) should be replaced by:

$$
Z_{m}(x)=\sin m x+\sum_{k=1}^{\infty} q^{k} s_{k}(x) .
$$

In this case, we have:

$$
r_{2}=1-q-\frac{q^{2}}{8}+\ldots
$$

In any case, the functions $c_{k}(x)$ and $s_{k}(x)$ are linear combinations of cosines and sines, respectively.

At this point, we should underline that (5) converges for $|q|<1$, so that the standard harmonic balance is only applicable in this region.

\subsection{An algebraic harmonic balance method}

In the Introduction, we have mentioned the existence of four discrete sets of characteristic values. After replacing these characteristic values for $r$ in (3), we have a periodic solution for equation (3). These characteristic values depend on $q$ and are labelled by an index. The former of these series, $r_{2 s}(q)$, has even index $2 s$ and the second, $r_{2 s+1}(q)$, has an odd index $2 s+1$, with $s=0,1,2, \ldots$ For each of these values of $s$, we have one even solution of the form:

$$
\begin{array}{r}
Y_{2 s}(x)=\sum_{k=0}^{\infty} A_{2 k} \cos (2 k x), \\
Y_{2 s+1}(x)=\sum_{k=0}^{\infty} A_{2 k+1} \cos (2 k+1) x .
\end{array}
$$

There are two more series of solutions which are odd. Their characteristic values are different from those corresponding to even solutions and we denote 
them as $r_{2 s}^{\prime}(q)$ and $r_{2 s+1}^{\prime}(q)$. Again, we have $s=0,1,2, \ldots$ These odd solutions are

$$
\begin{array}{r}
Z_{2 s}(x)=\sum_{k=1}^{\infty} B_{2 k} \sin (2 k x), \\
Z_{2 s+1}(x)=\sum_{k=0}^{\infty} B_{2 k+1} \sin (2 k+1) x .
\end{array}
$$

It is our goal the determination of approximate expressions for these solutions with a reasonable accuracy. In order to achieve it, we make use of our modified harmonic balance method, that we introduce here.

Probably, the best way to explain our method is by an illustration. To this end, let us choose for instance an odd index, hence we take $r_{2 s+1}(q)$. Any other choice would have been equally good. In this particular case, we know that the solution in terms of the eigenfunction is even and the period $p=2 \pi(11)$. Thus, let us find $r_{2 s+1}(q)$ and replace $Y_{2 s+1}(x)$ in (9) by:

$$
Y_{2 s+1}(x)=\sum_{k=0}^{n} P_{k}(q) \cos (2 k+1) x,
$$

where the coefficients $P_{k}(q)$ are polynomials of degree $k$ on $q$ to be determined. Thus, we are assuming from the very beginning that the series (12) are finite and sum from $k=0$ up to $k=n$. This Ansatz is in the core of our method and, therefore, no convergence conditions should be imposed on (12). Then, with the use of trigonometric relations, we obtain an expression of the following type:

$$
\sum_{j \text { odd }} f_{j} \cos j x=0
$$

with

$$
\begin{array}{r}
f_{1}=f_{1}\left(P_{0}, P_{1}\right), f_{3}=f_{3}\left(P_{0}, P_{1}, P_{2}\right), \ldots, f_{2 n-1}=f_{2 n-1}\left(P_{n-2}, P_{n-1}, P_{n}\right), \\
f_{2 n+1}=f_{2 n+1}\left(P_{n-1}, P_{n}\right) .
\end{array}
$$


These functions are linear in their arguments. Note that $f_{j}$ vanishes identically for $j$ even.

Let us go back to (13) and note that for different values of $j$, the functions $\cos (j x)$ are linearly independent. This shows that the coefficients $f_{j}$ for $j$ odd must be zero. This gives a system of equations providing a recurrence relation between the polynomials $P_{k}(q)$, which is:

$$
\begin{array}{r}
P_{1}=\frac{1}{q}\left(r_{m}-1-q\right) P_{0}, \\
P_{k-1}=-P_{k+1}-\frac{1}{q}\left((2 k+1)^{2}-r_{m}\right) P_{k}, \quad k=1,2, \ldots, n-1, \\
P_{n-1}=\frac{1}{q}\left(r_{m}-(2 n+1)^{2}\right) P_{n} .
\end{array}
$$

In order to determine the $P_{k}(q)$ we can proceed in two ways. Either we fix $P_{0}$ and then determine all others through the recurrence relations up to $P_{n}$ or proceed inversely, giving a value to $P_{n}$ and using the recurrence relations, we obtain the others. Let us use the latter. From $P_{n}$, we determine the coefficients $P_{k}, k=n-1, n-2, \ldots, 2,1$ by using (16). Note that the $P_{k}$ are polynomials in terms of $r_{m}$ and have a linear dependence on $P_{n}$, to be determined later. If we replace $P_{0}$ and $P_{1}$ on (15), we finally obtain an equation of the type:

$$
F\left(q, r_{m}\right)=0 .
$$

This function $F\left(q, r_{m}\right)$, which does not depend on $P_{n}$, is obviously a polynomial on $q$ and on $r_{m}$ with degree $n+1$ on the second variable. The solutions of (17) give $r_{m}$ as functions of $q, r_{m}=r_{m}(q)$. Since (17) is an algebraic equation, their solutions have to be obtained by numerical methods, this being the only numerical procedure in all the derivation. Among all solutions of (17), we have to choose one. Although $r_{m}$ may be either positive or negative, when $q=0$ in (3) one arrives to the harmonic oscillator equation, so that $r_{m}(0)=m^{2}$.

Thus, for each given value of $q$, we have obtained the coefficients $P_{k}(q)$, $k=0,1,2, \ldots, n-1$ in terms of $P_{n}(q)$ and the algebraic function $r_{m}(q)$. Once we have fixed $P_{n}(q)$, we have obtained an approximate analytical solution for the Mathieu equation (3). 
Let us insist on an important point: Contrarily to the usual harmonic balance method, we do not use any span of $r_{m}(q)$ in Taylor series.

We have already mentioned that when $q \longmapsto 0$, then, $Y_{m}(x) \longmapsto \cos m x$. This will be a condition on the choice of $P_{n}(q)$ once the value of $n$ has been fixed. Our choice for $m$ odd, $m=2 s+1$ is

$$
P_{n}(q):=q^{n-s} .
$$

This gives the correct limit as $q \longmapsto 0$.

Note that the precision of this approximation depends on $n$. There is no general rule to fix the value of this precision, but we can estimate the error. To this end, one possibility is the use of the following error parameter:

$$
E_{r}(n):=\frac{\int_{0}^{2 \pi}\left(y^{\prime \prime}(x)+\left(r_{m}-2 q \cos 2 x\right) y(x)\right)^{2} d x}{\int_{0}^{2 \pi} y^{2}(x) d x} .
$$

This error parameter (19) gives us an estimation on how far are both $r_{m}$ and $y(x)$ from the exact characteristic value and its corresponding periodic eigenfunction of the Mathieu equation (3).

This procedure can be illustrated by an example. Let us choose $m=1$ and $n=5$. Then, $F\left(q, r_{1}\right)$ as in (17) takes the following form:

$$
F\left(q, r_{1}\right)=\sum_{k=0}^{6} \alpha_{k}(q) r_{1}^{k}(q)
$$

where the coefficients $\alpha_{k}(q)$ are polynomials on $q$, which for our choice of the parameters give:

\section{TABLE 1}

This gives for the eigenfunction the following expression:

$$
y(x)=c \sum_{k=0}^{5} P_{k}\left(q, r_{1}\right) \cos (2 k+1) x,
$$

where $c$ is a normalization constant so that $y(0)=1$. The coefficients $P_{k}\left(q, r_{1}\right)$ are given by:

TABLE 2 
Let us go back to (20), choose a value of $q$ and write the final expression equal to zero. This is an algebraic equation which gives $r_{1}$. Note that we need to give the determination of the branch of the root to fix $r_{1}$. We choose the branch which gives the value one when $q=0$. Then, use this $r_{1}$ in the equation (22) right below, to obtain the normalization constant $c$, which is given by:

$$
c=\left[\sum_{k=0}^{5} b_{k}(q) r_{1}^{k}\right]^{-1}
$$

with

\section{TABLE 3}

In order to determine the characteristic value $r_{m}$ for a given value of $q$, we have to solve (17) with the choice $r_{m}(0)=m^{2}$. For instance, if $q=5$, $m=1$ and $r_{1}(0)=1$, we obtain $r_{1}=1.8581875415477507$. We approximate this value by the rational number $r_{1}=\frac{13}{7}$. In fact, the rational value $r_{1}$ has been chosen to give an error less that $0.05 \%$ with respect to the decimal solution obtained numerically. The approximate eigenfunction for $r_{1}$ takes the following form:

$$
\begin{gathered}
y(x)=\frac{104}{35} \cos x-\frac{32}{13} \cos 3 x+\frac{49}{90} \cos 5 x \\
-\frac{5}{86} \cos 7 x+\frac{19}{5159} \cos 9 x-\frac{1}{6469} \cos 11 x .
\end{gathered}
$$

This is an approximation to the Mathieu cosine function for the particular case $q=5$ and $m=1$. In the eigenfunction (23), we have used rational approximations for the coefficients. The procedure is the same as above; starting from the numerical value for $r_{1}$, we obtain numerical values for the coefficients of the trigonometric polynomial (23). Then, we approximate these numerical values by the rational numbers. These rational approximations give an error smaller than $0.05 \%$ in relation to the decimal expressions for the coefficients. From (18), we obtain the error $E_{r}(5)=4 \times 10^{-8}$.

We have seen in this example an important fact that shows the interest of our method: how if we take a short number of terms in the span (12), we get a surprisingly high level of accuracy, for a value of $q$ which is not small. 


\subsection{Some results.}

Let us define the error $e_{r}$ as $E_{r}$ in (19) multiplied by the integral of $y^{2}(x)$, i.e.,

$$
e_{r}(n):=\int_{0}^{2 \pi}\left(y^{\prime \prime}(x)+\left(r_{m}-2 q \cos 2 x\right) y(x)\right)^{2} d x
$$

In the following table, we compare the errors $E_{r}$ and $e_{r}$ for several values of $q$ and their corresponding values of $r_{1}$ when the highest order polynomial $P_{n}(q)$ has degrees $n=3,5$ and 40 . These approximations are expected to provide different values of $r_{1}$ which we shall denote as $r_{1, n}$. Note that the values of $r_{1, n}$ coincide for small values of $q$. These errors are smaller for higher values of $n$. We represent as rational numbers the values for $r_{1, n}$ that are the same for $r_{1,3}, r_{1,5}$ and $r_{1,40}$. The table is the following:

\section{TABLE 4}

We have written in italics the numbers for which the coincidence of the values $r_{1, n}$ with $r_{1,40}$ fail. After this failure, the value for the error $e_{r}$ increases abruptly. These numbers also show something quite interesting: for these values for which $e_{r}$ increases quickly or even abruptly, the values $E_{r}$ show a moderate increase. This suggest that $E_{r}$ is not a good error bound. One also concludes that $r_{n}$ is not very sensitive to the value $n$. On the other hand, the eigenfunction is expected to be quite sensitive to $n$, since the error $e_{r}$ is highly sensitive to $n$.

The comparison between the values of $r_{1,5}$ and $r_{1,40}$ shows that the choice $r_{1,5}$ gives a reasonable approximation. In Figure 1 , we plot $r_{1}(q)$ for the values $n=5,10,50$. Observe that these three curves are indistinguishable within the chosen precision.

\section{FIGURE 1}

If the index $m$ is even, all the above discussion remains valid although equation (12) should be replaced by

$$
Y_{2 s}(x)=\sum_{k=0}^{n} P_{k}(q) \cos (2 k) x
$$


Analogously, in order to have $Y_{m}(x) \longmapsto \cos (m x)$ as $q \longmapsto 0$, the choice of the highest order polynomial should be $P_{n}(q)=q^{n-s}, s=1,2, \ldots, n$.

Finally, a similar treatment can be applied when we are looking for odd approximate solutions to (3). These solutions have the following form:

$$
\begin{array}{r}
Z_{2 s+1}(x):=\sum_{k=0}^{n} P_{k}(q) \sin (2 k+1) x, \quad s=0,1,2, \ldots, \\
Z_{2 s}(x):=\sum_{k=0}^{n} P_{k}(q) \sin (2 k x), \quad s=1,2, \ldots
\end{array}
$$

The procedure is here exactly the same as in the previous case. Again, in order that $Z_{m}(x) \longmapsto \sin m x$, as $q \longmapsto 0$, we must take $P_{n}(q)=q^{n-s}$, $s=1,2, \ldots, n$, for the highest order polynomial.

\section{Mathieu equation with complex potential}

In the usual harmonic balance method, characteristic values are determined as power series in terms of $q$, as in the first equation in (4). As was established in earlier publications (see (11) and references quoted therein), the radii of convergence of these series depends on the existence of double points, i.e., the existence of a repeated characteristic value, different from zero, in one of the series.

The multiplicity of the characteristic values can be determined by the multiplicity of the solutions of equation (17). We conjecture that, when $q$ is real, the roots of (17) are all simple, so that double points should not exists. This is not the case when $q$ is purely imaginary, i.e., $q=i s$, as shown in $(11 ; 12)$. The relevance of the Mathieu equation with complex potential has been studied in $(16 ; 17)$.

Let us analyze the case $q=i s$. It is now convenient to use the notation $r_{m}(s)$ instead of $r_{m}(q)$ in order not to bring the imaginary unit everywhere.

In the present literature, it is established that double points appear for $m$ even only. Thus, let us start with the most simple even values of $m$, which obviously are $m=0$ and $m=2$. In any case, we always have that if $s=0$, $r_{m}(0)=m^{2}$.

Next, we determine $r_{0}(s)$ and $r_{2}(s)$ using (17). These functions are increasing and decreasing on $s$, respectively, for positive values of $s$ under the 
condition $r_{0}(0)<r_{2}(0)$. Therefore, it must exist a value of $s$ that we denote as $s_{b}$ with $s_{b}>0$ such that

$$
r_{0}\left(s_{b}\right)=r_{2}\left(s_{b}\right)=r_{*} .
$$

The number $s_{b}$ is often called the branching value and defines the radius of convergence of the Taylor series for $m=0$ and $m=2$. The number $r_{*}$ is the critical value. From (28), we see that for $s=s_{b}$ we have a double point, which is $r_{*}$. For $s>s_{b}$ the roots of (17) are complex conjugate, $r_{0}(s)=r_{2}^{*}(s)$, where the superscript ${ }^{*}$ denotes complex conjugation.

This procedure can be extended to all even values of $m$ for which a branching value satisfying $r_{m}\left(s_{b}\right)=r_{m+2}\left(s_{b}\right)$ must exist. In order to determine the double points, we replace $q$ by $i s$ in (17) and then we obtain the double roots. These double roots must obey the following pair of equations:

$$
F(i s, r)=0, \quad \frac{\partial}{\partial r} F(i s, r)=0 .
$$

Let us give a example of our results for $n=30$ (see also Figure 2). With the aid of equations (29), we can determine numerically the values of double points as well as their respective branching values. In this case, we have 9 double points that we shall label as $r_{*, k}, k=1,2, \ldots, 9$ being their respective branching values denoted as $s_{b, k}$.

\section{FIGURE 2}

The obtained values of $r_{*, k}$ and $s_{b, k}$ are given in the following table. It is interesting to remark that these results are similar to those obtained by C.H. Ziener et al. in (12):

\section{TABLE 5}

The use of least squares allows the approximation of $r_{*, k}, s_{b, k}$ by the following formulas:

$$
r_{*, k}=\frac{35}{8}-\frac{65}{4} k+\frac{419}{30} k^{2}, \quad\left|s_{b, k}\right|=\frac{14}{5}-\frac{19}{2} k+\frac{49}{6} k^{2} .
$$

As a final remark, we should stress that although our method does not consider power series on $q$, starting from the equation $F(q, r)=0$, it allows 
to estimate the radius of convergence of the power series on $q$ used in other approaches.

\section{Second method: Least squares}

Let us go back to equation (2), with the assumption that $f(x)$ is a periodic function with period $T$, which without loss of generality we can fix $T=2 \pi$. We also assume that $f(x)$ has a fixed parity either even or odd and, in addition, that $f(x)$ will depend on one or more real parameters $\alpha$, $\beta$, etc, as well as the characteristic value $r$. The characteristic value has to be determined through the periodicity conditions $y(0)=y(2 \pi)$ and $y^{\prime}(0)=$ $y^{\prime}(2 \pi)$.

As we did in the previous case, described in section 2, we do not introduce our method in its full generality. A simpler version will be much more illustrative and is given in a form such that its generalization will become obvious. In this spirit, let us assume that in (1), $B_{m}=0, m=1,2, \ldots$ Then, equation (2) expressed in the form (1) has an even solution that can be written in terms of cosine Fourier series:

$$
y(x)=\sum_{k=0}^{\infty} a_{k} \cos (k x) .
$$

Since we are looking for approximate solutions that could be handled in a computer, let us truncate the series (32) and consider the following sum:

$$
y_{n}(x):=\sum_{k=0}^{n} a_{k} \cos (k x) .
$$

Now, the goal is the determination of the parameter (characteristic values to be consistent with the terminology of the previous case) $r$ as well as the Fourier coefficients $a_{k}, k=0,1, \ldots, n$. Then, let us replace (32) in the left hand side of (1) (or (2), where $f(x)$ is even and spanned into cosine Fourier series). Since (32) is not the solution, but an approximation to the solution (31), this replacement will not satisfy the equation, i.e., it cannot be equal to zero. Instead, we obtain an expression of the type ( $m$ and $n$ do not have to be equal):

$$
z_{m}(x)=\sum_{k=0}^{m} b_{k} \cos (k x)
$$


As (2) is a linear equation, the coefficients $b_{k}$ depend linearly of the $a_{j}$ :

$$
b_{k}=\sum_{j=1}^{n} b_{k j} a_{j} .
$$

Here, the coefficients $b_{k j}$ depend on the characteristic value $r$, of the parameters $\alpha, \beta$, etc as well as the Fourier coefficients $A_{n}(x)$ of $f(x)$. Since the sequence $\left\{y_{n}(x)\right\}$ converges to the exact solution $y(x)$ as $n \longmapsto \infty$, the sequence $\left\{z_{m}(x)\right\}$ must converge to zero as $m \longmapsto \infty$. One may expect that this would be a point-wise convergence, but dealing with Fourier series of square integrable functions over a period (in this case $2 \pi$ ), it is more convenient to use the convergence in the mean. From this point of view,

$$
E_{m}:=\int_{0}^{2 \pi}\left|0-z_{m}(x)\right|^{2} d x=\left\|z_{m}\right\|^{2} \longmapsto 0 \quad \text { as } \quad m \longmapsto \infty .
$$

The value $E_{m}$ could be considered the square error of the approximation given by (32). We need to minimize this error. Note that $E_{m}$ depends on the coefficients $a_{k}$ in (32), so that the condition for the minimum should be

$$
\frac{\partial E_{m}}{\partial a_{i}}=0, \quad i=0,1,2, \ldots, n .
$$

In order to solve system (36), we note that the Fourier coefficients $b_{k}$ and $E_{m}$ have to be related by an identity of the form (Bessel identity):

$$
E_{m}=K \sum_{k=0}^{m} b_{k}^{2},
$$

where $K>0$ is a normalization constant. From (36) and (37), one gets

$$
0=\frac{\partial E_{m}}{\partial a_{i}}=2 K \sum_{k=0}^{m} b_{k} \frac{\partial b_{k}}{\partial a_{i}}, \quad i=0,1,2, \ldots, n .
$$

Taking into account (34), equation (38) yields:

$$
0=\sum_{k=0}^{m} b_{k} b_{k i}=\sum_{j=0}^{n}\left(\sum_{k=0}^{m} b_{k i} b_{k j}\right) a_{j}=\sum_{j=0}^{n} s_{i j} a_{j},
$$

where the meaning of $s_{i j}$ is obvious. Now, observe that equations $(34,39)$ have a quadratic dependence on $r$. Then, the determinant of the matrix 
with entries $s_{i j}, \Delta\left(s_{i j}\right)$, vanish provided that the solutions for the $a_{i}$ in (39) be different from the trivial one. This determinant is a polynomial function on $r$ :

$$
F(\alpha, \beta, \ldots, r)=\sum_{k=1}^{p} c_{k}\left(\alpha, \beta, \ldots, A_{r}, \ldots\right) r^{k},
$$

where $A_{r}$ are the Fourier coefficients of $f(x)$ in $(1)$. The roots of the polynomial $F=0$, where $F$ is as in (40), determine the characteristic values of $r$. Then, let us choose an eigenvalue of the matrix with entries $\left\{s_{i j}\right\}$ in order to obtain the Fourier coefficients $a_{k}$, which determine one approximate solution. The algebraic system in (39) is homogeneous; therefore, we need fixing one of the $a_{i}$ in the right side of (39), which has to be chosen depending on the properties of the solution we are looking for. For instance, in the Mathieu equation if $q \longmapsto 0$, then, the solution $y(x) \longmapsto \cos m x$. This condition give rise the analogous of equation (20).

We have implemented this second method in the Mathieu equation $(1,2)$, where the approximate solution is

$$
y_{n}(x)=\sum_{k=s}^{n} a_{k} \cos k x .
$$

In order to obtain periodic solutions with period $2 \pi$, we have to choose $s=1$ and $a_{1}=1$. If we want solutions with period $\pi$, then we should choose $s=0$ and $a_{0}=1$. The results that we have obtained matched with those obtained in Sections 2 and 3.

\subsection{On the asymptotic equivalence between both methods.}

As the title of this subsection suggests, we are going to show the equivalence between the two methods discussed in the present paper in the limit when $n$ goes to infinity, at least when applied to the Mathieu equation.

Thus, let us consider the Mathieu equation (2) with $q$ real and go back to equation (17). The function $F(q, r)$ has different forms depending on whether we are using our modified harmonic balance method or the least squares method. For the former and due to the fact that $F(q, r)$ is a polynomial of degree $n+1$, one has:

$$
F_{M H B}(q, r)=\prod_{k=0}^{n+1}\left(r-r_{k}(q)\right)
$$


Here, the roots are always real and their values should depend on the numerical precision in which they are computed. In the numerical experiments we have performed, we have systematically observed that the biggest root is spurious. This behavior is a consequence of the truncation of the Fourier series.

In the case of the least squares method, numerical experiments show that

$$
F_{L S}=\prod_{k=0}^{n}\left(r-R_{k}(q)\right)\left(r-R_{k}^{*}(q)+i \varepsilon_{k}\right),
$$

where we now denote the roots as $R_{k}(q)$. There is, however, an important difference, which is that our results give a non vanishing imaginary part for $R_{k}(q)$, so that $R_{k}(q)=\operatorname{Re}\left[R_{k}(q)\right]+i \operatorname{Im}\left[R_{k}(q)\right]$, with $\operatorname{Im}\left[R_{k}(q)\right] \neq 0$. In any case, $\operatorname{Re}\left[R_{k}(q)\right] \approx r_{k}(q)$.

The values $\varepsilon_{k}$ are real, very small and go to zero as $n \longmapsto \infty$.

In our numerical experiments, we have observed these features:

i.) The imaginary part of $R_{k}(q)$ is much smaller than its real part, so that

$$
\frac{\left|\operatorname{Im}\left[R_{k}(q)\right]\right|}{\left|\operatorname{Re}\left[R_{k}(q)\right]\right|}<<1,
$$

ii.) The imaginary part $\operatorname{Im}\left[R_{k}(q)\right]$ is small and can only appear as we increment the numerical precision, or in other words, when the number of significative digits is large. Nevertheless, $\operatorname{Im}\left[R_{n}(q)\right] \longmapsto 0$ when $n \longmapsto \infty$.

iii.) Observe the presence of the term $\varepsilon_{k}$ in (43). This goes to zero as $n$ increases.

iv.) From all the above, we conjecture the following result:

$$
\lim _{n \mapsto \infty}\left[r_{n}(q)-R_{n}(q)\right]=0 .
$$

The final conclusion is that for infinite precision (infinite number of significative digits) and in the limit $n \longmapsto \infty$, we have

$$
F_{L S}(q, r)=\prod_{k=0}^{\infty}\left(r-R_{k}(q)\right)^{2},
$$

and 


$$
F_{L S}(q, r)=\left[F_{M H B}(q, r)\right]^{2} .
$$

Therefore, when $n \longmapsto \infty$ both methods are equivalent and should give the same results with high precision and $n$ large. Nonetheless, we have already remarked that our modified harmonic balance is simpler to use as only requires to solve numerically an algebraic equation. Furthermore, this algebraic equation is much simpler than the equation that we need to solve with the least squares method.

Finally, we should remark that the above argument is applicable to the Mathieu equation only. However, our numerical experiments suggest that these results and particularly (47) are more general.

\section{Concluding remarks}

We have compared on the Mathieu equation two methods which should be valid to obtain analytic approximations for periodic solutions of Hill type equations. In the former, we have developed a modification of the harmonic balance method, based in the replacement of the coefficients of the Fourier series of solutions by polynomials, $P_{n}(q)$, on a parameter, here called $q$. This method has been shown to be quite simple and easy to implement, which is a good advantage with respect to other methods. In the case of the Mathieu equation, $q$ is a parameter characteristic of the equation (do not confuse with the characteristic value or eigenvalue, $r$ ) and could be either real or purely imaginary. The polynomials $P_{n}(q)$ can be determined by a recurrence formula.

Although the modified harmonic balance method is applicable to linear differential equations with periodic solutions, including the Hill equations, the first objective has been the evaluation of the characteristic values and periodic approximate solutions of the Mathieu equation. Our method provides these values in terms of a polynomial equation which also depends on $q$, so that the characteristic values $r$ depend on $q$, as expected. One of the advantages of the method, in relation to others valid only for small values of $q$, is that it is applicable to large values of $q$ (for instance, the standard harmonic balance relies on a Taylor span of $r(q)$ with a finite radius of convergence $(|q|<1$ in the studied examples), a procedure that we do not use here). 
We have obtained some reference numerical results to check the efficiency of the method and show that it is also applicable to purely imaginary values of $q$.

In addition, we have compared our results with those which can be obtained with the least squares method. It is noteworthy that our method is much simpler and gives better accuracy using a minor degree of precision. On the other hand, both methods seem to be equivalent in the limit when the number of terms in the approximation series go to infinity. This has been tested for the Mathieu equation, but our numerical experiments with other equations show that this result might be more general.

Thus, we have checked that our method works well and it is simpler and more efficient than others that look for approximate periodic solutions of linear ordinary differential equations. The next task will be to apply the method to higher order Hill equations with interest in Physics.

Finally one more remark. The harmonic balance method has been used to obtain approximations of periodic solutions of some non-linear ordinary differential equations $(2 ; 18)$. The possibility of a similar use for our modified harmonic balance is, in principle, possible, but very difficult as we have realized after some preliminary results.

\section{Acknowledgements}

Partial financing support is acknowledged to the Spanish MINECO (Project MTM2014-57129), the Junta de Castilla y León (Project GR224) and the Project ING 19/ i 402 of the Universidad Nacional de Rosario.

[1] M. Farkas, Periodic Motions (Springer, New York, 1994).

[2] A. Beléndez, A. Hernándes, T. Beléndez, M.L. Alvarez, S. Gallego, M. Ortuno, C. Neipp, Application of the harmonic balance method to a non-linear oscillateor typified by a mass attached to a stretched wire, J. Sound Vibr., 302, 1018-1029 (2007).

[3] Y.M. Chen, J.K. Liu, A new method based on the harmonic balance method for non-linear oscillators, Phys. Lett. A, 368, 371-378 (2007).

[4] J.D. García-Saldaña, A. Gasull, The period function and the Harmonic Balance Method, Bull. Scie. Math., 139, 33-60 (2015). 
[5] J. Meixner, F. W. Schäfke, G. Wolf, Mathieu Functions and Spheroidal Functions and their Mathematical Foundations: Further Studies (Springer, Berlin, 1980).

[6] K.M. Urwin, F.M. Arscott, Theory of the Whittaker-Hill Equation, Proc. Roy. Soc. Edinbourgh, 69, 28-44 (1970).

[7] D. Zwillinger, Handbook of Differential Equations (Academic, Boston MA, 1977).

[8] W. Magnus, S. Winkler, Hill's Equation (Dover, New York, 1979).

[9] M. Gadella, J. Negro, L.M. Nieto, G.P. Pronko, Two charged particles in the plane under a constant perpendicular magnetic field, Int. J. Theor. Phys., 50 , 2019-2028 (2011).

[10] N. W. MacLachlan, Theory and Applications of Mathieu Functions, (Oxford U.P., U.K., 1951).

[11] G. Blanch, D.S. Clemm, The double points of Mathieu's differential equation, Math. Comp., 23, 97-108 (1969).

[12] C.H. Ziener, M. Rückl, T. Kampf, W.R. Bauer, H.P. Schlemmer, Mathieu functions for purely imaginary parameters, Journal of Computational and Applied Mathematics 236, 4513-4524 (2012).

[13] P. Shivakumara, Jungong Xueb, On the double points of a Mathieu equation, Journal of Computational and Applied Mathematics 107, 111125 (1999).

[14] C.A. Dartora, K.Z. Nobrega, H.E. Hernandez-Figueroa, New analytical approximations for the Mathieu functions, Applied Mathematics and Computation 165, 447-458 (2005).

[15] D. Frenkel, R. Portugal, Algebraic methods to compute Mathieu functions, Journal of Physics A: Mathematical and General 34, 3541-3551 (2001).

[16] A. Fring, E2-quasi-exact solvability for non-Hermitian models, J. Phys A: Math. Theor., 48, 145301 (2015). 
[17] C. H. Ziener, F.T. Kurz, T. Kampf, Free induction decay caused by a dipole field, Phys. Rev. E, 91, 032707 (2015).

[18] M. Ghadimi, H.D. Kaliji, Application of the Harmonic Balance Method on Nonlinear Equations, World Applied Sciences Journal, 22, 532-537 (2013).

\section{TABLE 1}

$$
\begin{aligned}
& \alpha_{0}(q)=108056025+108056025 q-12612933 q^{2}-606708 q^{3}+14230 q^{4}+179 q^{5}-q^{6}, \\
& \alpha_{1}(q)=-128816766-20760741 q+1616980 q^{2}+37548 q^{3}-604 q^{4}-3 q^{5}, \\
& \alpha_{2}(q)=21967231+1206490 q-64222 q^{2}-700 q^{3}+6 q^{4}, \\
& \alpha_{3}(q)=-1234948-28458 q+980 q^{2}+4 q^{3} \\
& \alpha_{4}(q)=28743+285 q-5 q^{2}, \\
& \alpha_{5}(q)=-286-q \\
& \alpha_{6}(q)=1 .
\end{aligned}
$$




\section{TABLE 2}

$$
\begin{aligned}
P_{0}= & -108056025+606708 q^{2}-179 q^{4}+\left(20760741-37548 q^{2}+3 q^{4}\right) r_{1} \\
& +\left(-1206490+700 q^{2}\right) r_{1}^{2}+\left(28458-4 q^{2}\right) r_{1}^{3}-285 r_{1}^{4}+r_{1}^{5} \\
P_{1}= & 12006225 q-14051 q^{3}+q^{5}+\left(-972724 q+422 q^{3}\right) r_{1}+\left(25974 q-3 q^{3}\right) r_{1}^{2}-276 q r_{1}^{3}+q r_{1}^{4} \\
P_{2}= & -480249 q^{2}+170 q^{4}+\left(19699 q^{2}-2 q^{4}\right) r_{1}-251 q^{2} r_{1}^{2}++q^{2} r_{1}^{3} \\
P_{3}= & 9801 q^{3}-q^{5}-202 q^{3} r_{1}+q^{3} r_{1}^{2} \\
P_{4}= & -121 q^{4}+q^{4} r_{1} \\
P_{5}= & q^{5}
\end{aligned}
$$

\section{TABLE 3}

$$
\begin{aligned}
& b_{0}(q)=-108056025+12006225 q+126459 q^{2}-4250 q^{3}-130 q^{4}+q^{5} \\
& b_{1}(q)=20760741-972724 q-17849 q^{2}+220 q^{3}+2 q^{4} \\
& b_{2}(q)=-1206490+25974 q+449 q^{2}-2 q^{3} \\
& b_{3}(q)=28458-276 q-3 q^{2} \\
& b_{4}(q)=-285+q \\
& b_{5}(q)=1
\end{aligned}
$$




\section{TABLE 4}

\begin{tabular}{|c|c|c|c|c|c|c|c|c|}
\hline$q$ & .01 & .1 & .5 & 1. & 5. & 10. & 15. & 20. \\
\hline$r_{1,3}$ & $\frac{102}{101}$ & $\frac{89}{81}$ & $\frac{22}{15}$ & $\frac{93}{50}$ & 1.85826 & -2.39492 & -8.07144 & -14.3927 \\
\hline$E r$ & $10^{-17}$ & $3.10^{-11}$ & $6.10^{-11}$ & $2.10^{-8}$ & $5.10^{-3}$ & 0.4 & 3. & 9. \\
\hline$e r$ & $9.10^{-17}$ & $9.10^{-11}$ & $2.10^{-10}$ & $7.10^{-8}$ & $3.10^{-1}$ & $5.10^{2}$ & $10^{6}$ & $10^{5}$ \\
\hline$r_{1,5}$ & $\frac{102}{101}$ & $\frac{89}{81}$ & $\frac{22}{15}$ & $\frac{93}{50}$ & 1.85819 & -2.39914 & -8.10111 & -14.4912 \\
\hline$E r$ & $3.10^{-17}$ & $3.10^{-11}$ & $6.10^{-11}$ & $3.10^{-12}$ & $4.10^{-8}$ & 4. $10^{-10}$ & $10^{-3}$ & $10^{-2}$ \\
\hline er & $9.10^{-17}$ & $9.10^{-11}$ & $2.10^{-10}$ & $1.10^{-11}$ & $2.10^{-6}$ & .04 & 15 & $2.10^{3}$ \\
\hline$r_{1,40}$ & $\frac{102}{101}$ & $\frac{89}{81}$ & $\frac{22}{15}$ & $\frac{93}{50}$ & 1.85819 & -2.39914 & -8.10111 & -14.4913 \\
\hline$E r$ & $10^{-17}$ & $3.10^{-11}$ & $6.10^{-11}$ & $3.10^{-12}$ & $2.10^{-14}$ & $3.10^{-13}$ & $3.10^{-14}$ & $10^{-13}$ \\
\hline$e r$ & $8.10^{-17}$ & 1. $10^{-10}$ & $2.10^{-10}$ & $5.10^{-8}$ & $5.10^{-12}$ & $3.10^{-10}$ & $7.10^{-10}$ & 1. $10^{-8}$ \\
\hline
\end{tabular}

TABLE 5

$\begin{array}{lllll}k & 1 & 2 & 3 & 4\end{array}$

$\begin{array}{lllll}s_{b, k} & \pm 1.4688 & \pm 16.471 & \pm 47.806 & \pm 95.475\end{array}$

$\begin{array}{lllll}r_{*, k} & 2.0887 & 27.319 & 80.658 & 162.11\end{array}$

$\begin{array}{lllll} & \ldots & \cdots & \cdots & \cdots\end{array}$

$\begin{array}{lllllll}k & 5 & 6 & 7 & 8 & 9\end{array}$

$\begin{array}{llllll}s_{b, k} & \pm 159.48 & \pm 239.82 & \pm 336.49 & \pm 449.50 & \pm 578.84\end{array}$

$\begin{array}{llllll}r_{*, k} & 271.67 & 409.33 & 575.11 & 769.00 & 991.00\end{array}$ 


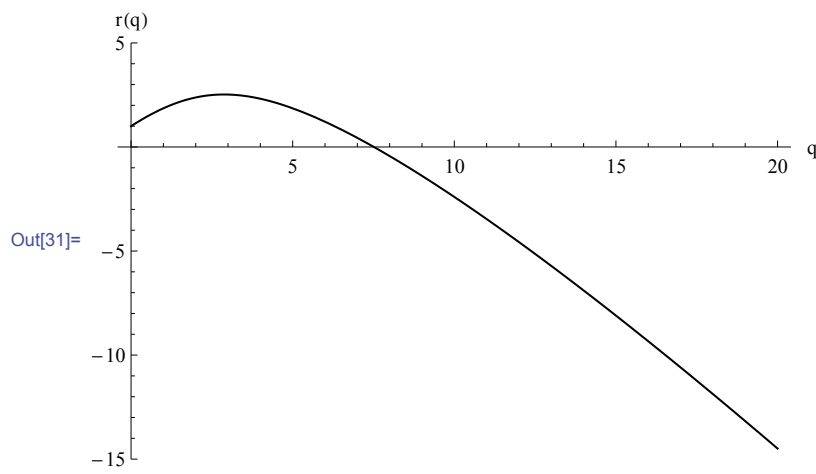

Figure .1: Dependence on $q$ of $r(q)$ for $m=1$ and $n=5,10,50$. Note that these three curves look like just one within the precision used.

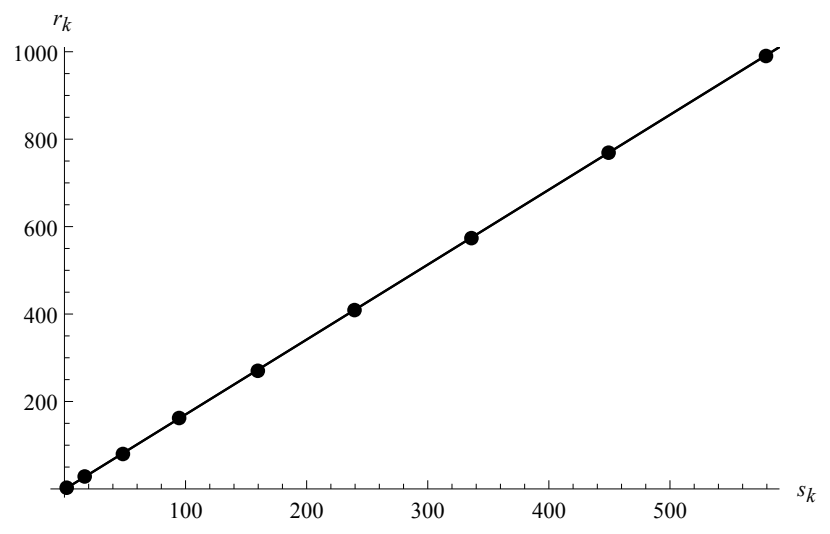

Figure .2: Localization of double points for $n=30$. The straight line is the interpolation curve. 